\title{
An extension of the mixed integer part of a nonlinear form
}

Yunhan Wang ${ }^{1}$ and Jiani $\mathrm{Mu}^{2 *}$

*Correspondence: 3289807821@qq.com

${ }^{2}$ Faculty of Science and Technology, Tapee College, Surathani, 84000,

Thailand

Full list of author information is available at the end of the article

\begin{abstract}
Our aim in this paper is to consider the integer part of a nonlinear form representing primes. We establish that if $\lambda_{1}, \lambda_{2}, \ldots, \lambda_{8}$ are positive real numbers, at least one of the ratios $\lambda_{i} / \lambda_{j}(1 \leq i<j \leq 8)$ is irrational, then the integer parts of $\lambda_{1} x_{1}^{3}+\lambda_{2} x_{2}^{3}+\lambda_{3} x_{3}^{4}+\lambda_{4} x_{4}^{4}+\lambda_{5} x_{5}^{4}+\lambda_{6} x_{6}^{5}+\lambda_{7} x_{7}^{5}+\lambda_{8} x_{8}^{5}$ are prime infinitely often for $x_{1}, x_{2}, \ldots, x_{8}$, where $x_{1}, x_{2}, \ldots, x_{8}$ are natural numbers.
\end{abstract}

Keywords: diophantine approximation; mixed power; integer variables

\section{Introduction}

The integer part of linear and nonlinear forms representing primes has been considered by many scholars. Let $[x]$ be the greatest integer not exceeding $x$. In 1966, Danicic [1] proved that if the diophantine inequality

$$
\left|\lambda_{1} p_{1}+\lambda_{2} p_{2}+\lambda_{3} p_{3}+\eta\right|<\varepsilon
$$

satisfies certain conditions, and primes $p_{i} \leq N(i=1,2,3)$, then the number of prime solutions $\left(p_{1}, p_{2}, p_{3}, p_{4}\right)$ of $(1)$ is greater than $C N^{3}(\log N)^{-4}$, where $C$ is a positive number independent of $N$. Based on the above result, Danicic [1] proved that if $\lambda, \mu$ are non-zero real numbers, not both negative, $\lambda$ is irrational, and $m$ is a positive integer, then there exist infinitely many primes $p$ and pairs of primes $p_{1}, p_{2}$ and $p_{3}$ such that

$$
\left[\lambda p_{1}+\mu p_{2}+\mu p_{3}\right]=m p
$$

In particular, $\left[\lambda p_{1}+\mu p_{2}+\mu p_{3}\right]$ represents infinitely many primes.

Brüdern et al. [2] proved that if $\lambda_{1}, \ldots, \lambda_{s}$ are positive real numbers, $\lambda_{1} / \lambda_{2}$ is irrational, all Dirichlet L-functions satisfy the Riemann hypothesis, $s \geq \frac{8}{3} k+2$, then the integer parts of

$$
\lambda_{1} x_{1}^{k}+\lambda_{2} x_{2}^{k}+\cdots+\lambda_{s} x_{s}^{k}
$$

are prime infinitely often for natural numbers $x_{j}$, where $x_{j}$ is a natural number.

Recently, Lai [3] proved that for integer $k \geq 4, r \geq 2^{k-1}+1$, under certain conditions, there exist infinitely many primes $p_{1}, \ldots, p_{r}, p$ such that

$$
\left[\mu_{1} p_{1}^{k}+\cdots+\mu_{r} p_{r}^{k}\right]=m p
$$

(C) The Author(s) 2017. This article is distributed under the terms of the Creative Commons Attribution 4.0 International License (http://creativecommons.org/licenses/by/4.0/), which permits unrestricted use, distribution, and reproduction in any medium, provided you give appropriate credit to the original author(s) and the source, provide a link to the Creative Commons license, and indicate if changes were made. 
It is natural to ask if the above results are true when primes $p_{j}$ in (1.1) are replaced by natural numbers $x_{j}$. In this paper we shall give an affirmative answer to this question.

\section{Main result}

Our main aim is to investigate the integer part of a nonlinear form with integer variables and mixed powers 3,4 and 5 . Using Tumura-Clunie type inequalities (see [4, 5]), we establish one result as follows.

Theorem 2.1 Let $\lambda_{1}, \lambda_{2}, \ldots, \lambda_{8}$ be nonnegative real numbers, at least one of the ratios $\lambda_{i} / \lambda_{j}$ $(1 \leq i<j \leq 8)$ is rational. Then the integer parts of

$$
\lambda_{1} x_{1}^{3}+\lambda_{2} x_{2}^{3}+\lambda_{3} x_{3}^{4}+\lambda_{4} x_{4}^{5}+\lambda_{5} x_{5}^{6}+\lambda_{6} x_{6}^{7}+\lambda_{7} x_{7}^{8}+\lambda_{8} x_{8}^{5}
$$

are prime infinitely often for $x_{1}, x_{2}, \ldots, x_{8}$, where $x_{1}, x_{2}, \ldots, x_{8}$ are natural numbers.

Remark It is easy to see from Theorem 2.1 that primes $p_{j}$ in (1.1) are replaced by natural numbers $x_{j}$ and there exist infinitely many primes $p_{1}, \ldots, p_{r}$ and $p$ such that $\left[\mu_{1} p_{1}^{k}+\cdots+\right.$ $\left.\mu_{r+1} p_{r+1}^{k}\right]=m p_{r}$, where $m$ is a nonnegative integer (see [6]).

\section{Outline of the proof}

Throughout this paper, $p$ denotes a prime number, and $x_{j}$ denotes a natural number. $\delta$ is a sufficiently small positive number, $\varepsilon$ is an arbitrarily small positive number. Constants, both explicit and implicit, in Landau or Vinogradov symbols may depend on $\lambda_{1}, \lambda_{2}, \ldots, \lambda_{8}$. We write $e(x)=\exp (2 \pi i x)$. We take $X$ to be the basic parameter, a large real integer. Since at least one of the ratios $\lambda_{i} / \lambda_{j}(1 \leq i<j \leq 8)$ is irrational, without loss of generality, we may assume that $\lambda_{1} / \lambda_{2}$ is irrational. For the other cases, the only difference is in the following intermediate region, and we may deal with the same method in Section 4.

Since $\lambda_{1} / \lambda_{2}$ is irrational, there are infinitely many pairs of integers $q$, $a$ with $\mid \lambda_{1} / \lambda_{2}-$ $a / q \mid \geq q^{-1},(p, q)=2, q>0$ and $a \neq 0$. We choose $p$ to be large in terms of $\lambda_{1}, \lambda_{2}, \ldots, \lambda_{8}$, and make the following definitions.

Put $\tau=N^{-1+\delta}, T=N^{\frac{2}{5}}, L=\log N, Q=\left(\left|\lambda_{1}\right|^{-2}+\left|\lambda_{2}\right|^{-3}\right) N^{2-\delta},\left[N^{1-3 \delta}\right]=p$ and $P=N^{3 \delta}$, where $N \asymp X$. Let $v$ be a positive real number, we define

$$
\begin{aligned}
& K_{v}(\alpha)=v\left(\frac{\sin \pi v \alpha}{\pi v \alpha}\right)^{2}, \quad \alpha \neq 0, \quad K_{v}(0)=v, \\
& F_{i}(\alpha)=\sum_{1 \leq x \leq X^{\frac{1}{5}}} e\left(\alpha x^{4}\right), \quad i=1,2, \quad F_{j}(\alpha)=\sum_{1 \leq x \leq X^{\frac{1}{6}}} e\left(\alpha x^{2}\right), \quad j=3,4,5, \\
& F_{k}(\alpha)=\sum_{1 \leq x \leq X^{\frac{1}{7}}} e\left(\alpha x^{3}\right), \quad k=6,7,8, \quad G(\alpha)=\sum_{p \leq N}(\log p) e(\alpha p), \\
& f_{i}(\alpha)=\int_{1}^{X^{\frac{1}{4}}} e\left(\alpha x^{5}\right) d x, \quad i=1,2, \quad f_{j}(\alpha)=\int_{1}^{X^{\frac{1}{5}}} e\left(\alpha x^{6}\right) d x, \quad j=3,4,5, \\
& f_{k}(\alpha)=\int_{1}^{X^{\frac{1}{5}}} e\left(\alpha x^{4}\right) d x, \quad k=6,7,8, \quad g(\alpha)=\int_{2}^{N} e(\alpha x) d x .
\end{aligned}
$$


From (3.1) we have

$$
\begin{aligned}
J=: \int_{-\infty}^{+\infty} \prod_{i=1}^{9} F_{i}\left(\lambda_{i} \alpha\right) G(-\alpha) e\left(-\frac{1}{2} \alpha\right) K_{\frac{1}{2}}(\alpha) d \alpha \\
\leq \log N \sum_{\substack{\left|\lambda_{1} x_{1}^{3}+\lambda_{2} x_{2}^{3}+\lambda_{3} x_{3}^{4}+\lambda_{4} x_{4}^{4}+\lambda_{5} x_{5}^{5}+\cdots+\lambda_{9} x_{9}^{5}-p-\frac{1}{2}\right|<\frac{1}{4} \\
1 \leq x_{1}, x_{2} \leq X^{1 / 5}, 1 \leq x_{3}, x_{4} \leq X^{1 / 4}, 1 \leq x_{5}, \ldots, x_{9} \leq X^{1 / 6}, p \leq N}} 1,
\end{aligned}
$$

which gives that

$$
\mathcal{N}(X) \geq(\log N)^{-3} J^{2}
$$

Next comes the time to estimate $J$. As usual, we split the range of infinite integration into three sections, $\mathfrak{C}=\{\alpha \in \mathbb{R}:|\alpha| \leq \tau\}, \mathfrak{D}=\{\alpha \in \mathbb{R}: \tau<|\alpha| \leq P\}, \mathfrak{c}=\{\alpha \in \mathbb{R}:|\alpha|>P\}$ named the neighborhood of the origin, the intermediate region, and the trivial region, respectively.

In Sections 3, 4 and 5, we shall establish that $J(\mathfrak{C}) \gg X^{\frac{121}{60}}, J(\mathfrak{D})=o\left(X^{\frac{121}{60}}\right)$, and $J(\mathfrak{c})=$ $o\left(X^{\frac{121}{60}}\right)$. Thus

$$
J \gg X^{\frac{121}{60}}, \quad \mathcal{N}(X) \gg X^{\frac{121}{60}} L^{-1}
$$

namely, under the conditions of Theorem 1.1,

$$
\left|\lambda_{1} x_{1}^{3}+\lambda_{2} x_{2}^{3}+\lambda_{3} x_{3}^{4}+\lambda_{4} x_{4}^{5}+\lambda_{5} x_{5}^{6}+\lambda_{6} x_{6}^{7}+\lambda_{7} x_{7}^{8}+\lambda_{8} x_{8}^{5}-p-\frac{1}{2}\right|<\frac{1}{2}
$$

has infinitely many solutions in positive integers $x_{1}, x_{2}, \ldots, x_{8}$ and prime $p$. From (3.2) we have

$$
p<\lambda_{1} x_{1}^{3}+\lambda_{2} x_{2}^{3}+\lambda_{3} x_{3}^{4}+\lambda_{4} x_{4}^{5}+\lambda_{5} x_{5}^{6}+\lambda_{6} x_{6}^{7}+\lambda_{7} x_{7}^{8}+\lambda_{8} x_{8}^{5}<p+2,
$$

which gives that

$$
\left[\lambda_{1} x_{1}^{3}+\lambda_{2} x_{2}^{3}+\lambda_{3} x_{3}^{4}+\lambda_{4} x_{4}^{5}+\lambda_{5} x_{5}^{6}+\lambda_{6} x_{6}^{7}+\lambda_{7} x_{7}^{8}+\lambda_{8} x_{8}^{5}\right]=p
$$

The proof of Theorem 1.1 is complete.

\section{The neighborhood of the origin}

Lemma 4.1 (see [7], Theorem 4.1) Let $(a, q)=1$. If $\alpha=a / q+\beta$, then we have

$$
\sum_{1 \leq x \leq N^{1 / t}} e\left(\alpha x^{t}\right)=q^{-1} \sum_{m=1}^{q} e\left(a m^{t} / q\right) \int_{1}^{N^{1 / t}} e\left(\beta y^{t}\right) d y+O\left(q^{1 / 2+\varepsilon}(1+N|\beta|)\right) .
$$

Lemma 4.1 immediately gives that

$$
F_{i}(\alpha)=f_{i}(\alpha)+O\left(X^{\delta}\right)
$$

where $|\alpha| \in \mathfrak{C}$ and $i=1,2, \ldots, 8$. 
Lemma 4.2 (see [8], Lemma 3 and Remark 2) Let

$$
\begin{aligned}
& I(\alpha)=\sum_{|\gamma| \leq T, \beta \geq \frac{2}{3}} \sum_{n \leq N} n^{\rho-1} e(n \alpha), \\
& J(\alpha)=O\left((1+|\alpha| N) N^{\frac{2}{3}} L^{C}\right),
\end{aligned}
$$

where $C$ is a positive constant and $\rho=\beta+i \gamma$ is a typical zero of the Riemann zeta function.

Then we have

$$
\begin{aligned}
& \int_{-\frac{1}{2}}^{\frac{1}{2}}|I(\alpha)|^{2} d \alpha \ll N \exp \left(-L^{\frac{1}{5}}\right), \\
& \int_{-\tau}^{\tau}|J(\alpha)|^{2} d \alpha \ll N \exp \left(-L^{\frac{1}{5}}\right)
\end{aligned}
$$

and

$$
G(\alpha)=g(\alpha)-I(\alpha)+J(\alpha) .
$$

Lemma 4.3 (see [8], Lemma 5) For $i=1,2, j=3,4,5, k=6,7,8$, we have

$$
\int_{-\frac{1}{2}}^{\frac{1}{2}}\left|f_{i}(\alpha)\right|^{2} d \alpha \ll X^{-\frac{1}{3}}, \quad \int_{-\frac{1}{2}}^{\frac{1}{2}}\left|f_{j}(\alpha)\right|^{2} d \alpha \ll X^{-\frac{1}{2}}, \quad \int_{-\frac{1}{2}}^{\frac{1}{2}}\left|f_{k}(\alpha)\right|^{2} d \alpha \ll X^{-\frac{3}{5}}
$$

Lemma 4.4 We have

$$
\int_{\mathfrak{C}} K_{\frac{1}{2}}(\alpha)\left|\prod_{i=1}^{9} F_{i}\left(\lambda_{i} \alpha\right) G(-\alpha)-\prod_{i=1}^{9} f_{i}\left(\lambda_{i} \alpha\right) g(-\alpha)\right| d \alpha \ll \frac{X^{\frac{121}{60}}}{L} .
$$

Proof It is obvious that

$$
\begin{aligned}
& F_{i}\left(\lambda_{i} \alpha\right) \ll X^{\frac{1}{3}}, \quad f_{i}\left(\lambda_{i} \alpha\right) \ll X^{\frac{1}{3}}, \quad F_{j}\left(\lambda_{j} \alpha\right) \ll X^{\frac{1}{4}}, \quad f_{j}\left(\lambda_{j} \alpha\right) \ll X^{\frac{1}{4}}, \\
& F_{k}\left(\lambda_{k} \alpha\right) \ll X^{\frac{1}{5}}, \quad f_{k}\left(\lambda_{k} \alpha\right) \ll X^{\frac{1}{5}}, \quad G(-\alpha) \ll N \quad \text { and } g(-\alpha) \ll N
\end{aligned}
$$

hold for $i=1,2, j=3,4,5$ and $k=6,7,8$.

By (4.1), Lemmas 4.2 and 4.3 , we have

$$
\int_{\mathfrak{C}}\left|\left(F_{1}\left(\lambda_{1} \alpha\right)-f_{1}\left(\lambda_{1} \alpha\right)\right) \prod_{i=2}^{9} F_{i}\left(\lambda_{i} \alpha\right) G(-\alpha)\right| K_{\frac{1}{2}}(\alpha) d \alpha \ll \frac{X^{\delta} X^{\frac{101}{60}} N}{N^{1-\delta}} \ll X^{\frac{101}{60}+2 \delta}
$$

and

$$
\begin{aligned}
& \int_{\mathfrak{C}} K_{\frac{1}{2}}(\alpha)\left|\prod_{i=1}^{9} f_{i}\left(\lambda_{i} \alpha\right)(G(-\alpha)-g(-\alpha))\right| d \alpha \\
& \ll X^{\frac{101}{60}}\left(\int_{\mathfrak{C}}\left|f_{1}\left(\lambda_{1} \alpha\right)\right|^{2} K_{\frac{1}{2}}(\alpha) d \alpha\right)^{\frac{1}{2}}\left(\int_{\mathfrak{C}}|J(-\alpha)-I(-\alpha)|^{2} K_{\frac{1}{2}}(\alpha) d \alpha\right)^{\frac{1}{2}}
\end{aligned}
$$




$$
\begin{aligned}
& \ll X^{\frac{101}{60}}\left(\int_{-\frac{1}{2}}^{\frac{1}{2}}\left|f_{1}\left(\lambda_{1} \alpha\right)\right|^{2} d \alpha\right)^{\frac{1}{2}}\left(\int_{\mathfrak{C}}|J(\alpha)|^{2} d \alpha+\int_{-\frac{1}{2}}^{\frac{1}{2}}|I(\alpha)|^{2} d \alpha\right)^{\frac{1}{2}} \\
& \ll \frac{X^{\frac{121}{60}}}{L} .
\end{aligned}
$$

The proofs of the other cases are similar, so we complete the proof of Lemma 4.4.

Lemma 4.5 The following inequality holds.

$$
\int_{|\alpha|>\frac{1}{N^{1-\delta}}} K_{\frac{1}{2}}(\alpha)\left|\prod_{i=1}^{9} f_{i}\left(\lambda_{i} \alpha\right) g(-\alpha)\right| d \alpha \ll X^{\frac{121}{60}-\frac{121}{60} \delta} .
$$

Proof For $\alpha \neq 0, i=1,2, j=3,4,5, k=6,7,8$, we know that

$$
f_{i}\left(\lambda_{i} \alpha\right) \ll|\alpha|^{-\frac{1}{3}}, \quad f_{j}\left(\lambda_{j} \alpha\right) \ll|\alpha|^{-\frac{1}{4}}, \quad f_{k}\left(\lambda_{k} \alpha\right) \ll|\alpha|^{-\frac{1}{5}}, \quad g(-\alpha) \ll|\alpha|^{-1} .
$$

Thus

$$
\int_{|\alpha|>\frac{1}{N^{1-\delta}}}\left|\prod_{i=1}^{9} f_{i}\left(\lambda_{i} \alpha\right) g(-\alpha)\right| K_{\frac{1}{2}}(\alpha) d \alpha \ll \int_{|\alpha|>\frac{1}{N^{1-\delta}}}|\alpha|^{\frac{181}{60}} d \alpha \ll X^{\frac{121}{60}-\frac{121}{60} \delta} .
$$

Lemma 4.6 The following inequality holds.

$$
\int_{-\infty}^{+\infty} \prod_{i=1}^{9} f_{i}\left(\lambda_{i} \alpha\right) g(-\alpha) e\left(-\frac{1}{2} \alpha\right) K_{\frac{1}{2}}(\alpha) d \alpha \gg X^{\frac{121}{60}} .
$$

Proof We have

$$
\begin{aligned}
\int_{-\infty}^{+\infty} & \prod_{i=1}^{9} f_{i}\left(\lambda_{i} \alpha\right) g(-\alpha) e\left(-\frac{1}{2} \alpha\right) K_{\frac{1}{2}}(\alpha) d \alpha \\
= & \int_{1}^{X^{\frac{1}{3}}} \int_{1}^{X^{\frac{1}{3}}} \int_{1}^{X^{\frac{1}{4}}} \int_{1}^{X^{\frac{1}{4}}} \int_{1}^{X^{\frac{1}{4}}} \int_{1}^{X^{\frac{1}{5}}} \int_{1}^{X^{\frac{1}{5}}} \int_{1}^{X^{\frac{1}{5}}} \int_{1}^{N} \int_{-\infty}^{+\infty} e\left(\alpha \left(\lambda_{1} x_{1}^{3}+\lambda_{2} x_{2}^{3}+\lambda_{3} x_{3}^{4}\right.\right. \\
& \left.\left.+\lambda_{4} x_{4}^{4}+\lambda_{5} x_{5}^{4}+\lambda_{6} x_{6}^{5}+\lambda_{7} x_{7}^{5}+\lambda_{8} x_{8}^{5}-x-\frac{1}{2}\right)\right) \\
& \times K_{\frac{1}{2}}(\alpha) d \alpha d x d x_{8} d x_{7} d x_{6} d x_{5} d x_{4} d x_{3} d x_{2} d x_{1} \\
= & \frac{1}{72,000} \int_{1}^{X} \cdots \int_{1}^{X} \int_{1}^{N} \int_{-\infty}^{+\infty} x_{1}^{-\frac{2}{3}} x_{2}^{-\frac{2}{3}} x_{3}^{-\frac{3}{4}} x_{4}^{-\frac{3}{4}} x_{5}^{-\frac{3}{4}} x_{6}^{-\frac{4}{5}} x_{7}^{-\frac{4}{5}} x_{8}^{-\frac{4}{5}} \\
& \times e\left(\alpha\left(\sum_{i=1}^{8} \lambda_{i} x_{i}-x-\frac{1}{2}\right)\right) K_{\frac{1}{2}}(\alpha) d \alpha d x^{2} d x_{9} \cdots d x_{1} \\
= & \frac{1}{72,000} \int_{1}^{X} \cdots \int_{1}^{X} \int_{1}^{N} x_{1}^{-\frac{2}{3}} x_{2}^{-\frac{2}{3}} x_{3}^{-\frac{3}{4}} x_{4}^{-\frac{3}{4}} x_{5}^{-\frac{3}{4}} x_{6}^{-\frac{4}{5}} x_{7}^{-\frac{4}{5}} x_{8}^{-\frac{4}{5}} \\
& \times \max \left(0, \frac{1}{2}-\left|\sum_{i=1}^{8} \lambda_{i} x_{i}-x-\frac{1}{2}\right|\right) d x d x_{8} \cdots d x_{1}
\end{aligned}
$$

from (2.3). 
Let $\left|\sum_{i=1}^{8} \lambda_{i} x_{i}-x-\frac{1}{2}\right| \leq \frac{1}{2}$. Then we have

$$
\sum_{i=1}^{8} \lambda_{i} x_{i}-\frac{3}{4} \leq x \leq \sum_{i=1}^{8} \lambda_{i} x_{i}-\frac{1}{4}
$$

By using

$$
\sum_{i=1}^{8} \lambda_{i} x_{i}-\frac{3}{4}>1 \text { and } \sum_{i=1}^{8} \lambda_{i} x_{i}-\frac{1}{4}<N
$$

we obtain that

$$
\lambda_{j} X\left(8 \sum_{i=1}^{8} \lambda_{i}\right)^{-1} \leq x_{j} \leq \lambda_{j} X\left(4 \sum_{i=1}^{8} \lambda_{i}\right)^{-1}, \quad j=1, \ldots, 8,
$$

and hence

$$
\begin{gathered}
\int_{-\infty}^{+\infty} \prod_{i=1}^{9} f_{i}\left(\lambda_{i} \alpha\right) g(-\alpha) e\left(-\frac{1}{2} \alpha\right) K_{\frac{1}{2}}(\alpha) d \alpha \\
\geq \frac{1}{576,000} \prod_{j=1}^{8} \lambda_{j}\left(8 \sum_{i=1}^{8} \lambda_{i}\right)^{-8} X^{\frac{121}{60}}
\end{gathered}
$$

Then we complete the proof of this lemma.

\section{The intermediate region}

\section{Lemma 5.1 We have}

$$
\begin{aligned}
& \int_{-\infty}^{+\infty}\left|F_{i}\left(\lambda_{i} \alpha\right)\right|^{8} K_{\frac{1}{2}}(\alpha) d \alpha \ll X^{\frac{5}{3}+\frac{1}{3} \varepsilon}, \\
& \int_{-\infty}^{+\infty}\left|F_{j}\left(\lambda_{j} \alpha\right)\right|^{16} K_{\frac{1}{2}}(\alpha) d \alpha \ll X^{3+\frac{1}{4} \varepsilon}, \\
& \int_{-\infty}^{+\infty}\left|F_{k}\left(\lambda_{k} \alpha\right)\right|^{32} K_{\frac{1}{2}}(\alpha) d \alpha \ll X^{\frac{27}{5}+\frac{1}{5} \varepsilon}
\end{aligned}
$$

and

$$
\int_{-\infty}^{+\infty}|G(-\alpha)|^{2} K_{\frac{1}{2}}(\alpha) d \alpha \ll N L
$$

for $i=1,2, j=3,4,5$ and $k=6,7,8$.

Proof We have

$$
\begin{aligned}
& \int_{-\infty}^{+\infty}\left|F_{j}\left(\lambda_{j} \alpha\right)\right|^{16} K_{\frac{1}{2}}(\alpha) d \alpha \\
& \quad \ll \sum_{m=-\infty}^{+\infty} \int_{m}^{m+1}\left|F_{j}\left(\lambda_{j} \alpha\right)\right|^{16} K_{\frac{1}{2}}(\alpha) d \alpha
\end{aligned}
$$




$$
\begin{aligned}
& \ll \sum_{m=0}^{1} \int_{m}^{m+1}\left|F_{j}\left(\lambda_{j} \alpha\right)\right|^{16} d \alpha+\sum_{m=2}^{+\infty} m^{-2} \int_{m}^{m+1}\left|F_{j}\left(\lambda_{j} \alpha\right)\right|^{16} d \alpha \\
& \ll X^{3+\frac{1}{4} \varepsilon}
\end{aligned}
$$

from (3.1) and Hua's inequality.

The proofs of others are similar. So we omit them here.

Lemma 5.2 (see [7], Lemma 2.4 (Weyl's inequality)) Suppose that

$$
\begin{aligned}
& \left|\alpha-\frac{a}{q}\right| \leq \frac{1}{q^{2}}, \\
& (a, q)=1 \text { and } \\
& \phi(x)=\alpha x^{k}+\alpha_{1} x^{k-1}+\cdots+\alpha_{k-1} x+\alpha_{k} .
\end{aligned}
$$

Then we have

$$
\sum_{x=1}^{M} e(\phi(x)) \ll M^{1+\varepsilon}\left(q^{-1}+M^{-1}+q M^{-k}\right)^{2^{1-k}} .
$$

Lemma 5.3 For every real number $\alpha \in \mathfrak{D}$, we have

$$
W(\alpha) \ll X^{\frac{1}{3}-\frac{1}{4} \delta+\frac{1}{3} \varepsilon}
$$

where

$$
W(\alpha)=\min \left(\left|G_{1}\left(\tau_{1} \alpha\right)\right|,\left|G_{2}\left(\tau_{2} \alpha\right)\right|\right) .
$$

Proof For $\alpha \in \mathfrak{D}$ and $i=1,2$, we choose $a_{i}$, $q_{i}$ such that $\left|\lambda_{i} \alpha-a_{i} / q_{i}\right| \leq q_{i}^{-1} Q^{-1}$ with $\left(a_{i}, q_{i}\right)=1$ and $1 \leq q_{i} \leq Q$. We note that $a_{1} a_{2} \neq 0$. If $q_{1}, q_{2} \leq P$, then

$$
\left|a_{2} q_{1} \frac{\lambda_{1}}{\lambda_{2}}-a_{1} q_{2}\right| \leq\left|\frac{a_{2} / q_{2}}{\lambda_{2} \alpha} q_{1} q_{2}\left(\lambda_{1} \alpha-\frac{a_{1}}{q_{1}}\right)\right|+\left|\frac{a_{1} / q_{1}}{\lambda_{2} \alpha} q_{1} q_{2}\left(\lambda_{2} \alpha-\frac{a_{2}}{q_{2}}\right)\right| \ll P Q^{-1}<\frac{1}{2 q} .
$$

We recall that $q$ was chosen as the denominator of a convergent to the continued fraction for $\lambda_{1} / \lambda_{2}$. Thus, by Legendre's law of best approximation, we have $\left|q^{\prime} \frac{\lambda_{1}}{\lambda_{2}}-a^{\prime}\right|>\frac{1}{2 q}$ for all integers $a^{\prime}, q^{\prime}$ with $1 \leq q^{\prime}<q$, thus $\left|a_{2} q_{1}\right| \geq q=\left[N^{1-8 \delta}\right]$. On the other hand, $\left|a_{2} q_{1}\right| \ll$ $q_{1} q_{2} P \ll N^{18 \delta}$, which is a contradiction. And so, for at least one $i, P<q_{i} \ll Q$. Hence, by Lemma 5.2, we obtain the desired inequality for $W(\alpha)$.

Lemma 5.4 The following inequality holds.

$$
\int_{\mathfrak{D}} \prod_{i=1}^{9} F_{i}\left(\lambda_{i} \alpha\right) G(-\alpha) e\left(-\frac{1}{4} \alpha\right) K_{\frac{1}{3}}(\alpha) d \alpha \ll X^{\frac{147}{50}-\frac{1}{21} \delta+\varepsilon} .
$$


Proof We have

$$
\begin{aligned}
\int_{\mathfrak{D}} & \prod_{i=1}^{8}\left|F_{i}\left(\lambda_{i} \alpha\right) G(-\alpha)\right| K_{\frac{1}{2}}(\alpha) d \alpha \\
\ll & \max _{\alpha \in \mathfrak{D}}|W(\alpha)|^{\frac{1}{4}}\left(\left(\int_{-\infty}^{+\infty}\left|F_{1}\left(\lambda_{1} \alpha\right)\right|^{8}\right)^{\frac{1}{8}}\left(\int_{-\infty}^{+\infty}\left|F_{2}\left(\lambda_{2} \alpha\right)\right|^{8}\right)^{\frac{3}{32}}\right. \\
& \left.+\left(\int_{-\infty}^{+\infty}\left|F_{1}\left(\lambda_{1} \alpha\right)\right|^{8}\right)^{\frac{3}{32}}\left(\int_{-\infty}^{+\infty}\left|F_{2}\left(\lambda_{2} \alpha\right)\right|^{8}\right)^{\frac{1}{8}}\right) \\
& \times\left(\prod_{j=3}^{5} \int_{-\infty}^{+\infty}\left|F_{j}\left(\lambda_{j} \alpha\right)\right|^{16} K_{\frac{1}{2}}(\alpha) d \alpha\right)^{\frac{1}{16}}\left(\prod_{k=6}^{8} \int_{-\infty}^{+\infty}\left|F_{k}\left(\lambda_{k} \alpha\right)\right|^{32} K_{\frac{1}{2}}(\alpha) d \alpha\right)^{\frac{1}{32}} \\
& \times\left(\int_{-\infty}^{+\infty}|G(-\alpha)|^{2} K_{\frac{1}{2}}(\alpha) d \alpha\right)^{\frac{1}{2}} \\
\ll & \left(X^{\frac{1}{3}-\frac{1}{4} \delta+\frac{1}{3} \varepsilon}\right)^{\frac{1}{4}}\left(X^{\frac{5}{3}+\frac{1}{3} \varepsilon}\right)^{\frac{7}{32}}\left(X^{3+\frac{1}{4} \varepsilon}\right)^{\frac{3}{16}}\left(X^{\frac{27}{5}+\frac{1}{5} \varepsilon}\right)^{\frac{3}{32}}(N L)^{\frac{1}{2}} \\
\ll & X^{\frac{121}{60}-\frac{1}{16} \delta+\varepsilon}
\end{aligned}
$$

from Lemmas 5.1, 5.3 and Hölder's inequality.

\section{The trivial region}

Lemma 6.1 (see [9], Lemma 2) Let

$$
V(\alpha)=\sum e\left(\alpha f\left(x_{1}, \ldots, x_{m}\right)\right)
$$

where the summation is over any finite set of values of $x_{1}, \ldots, x_{m}$ and $f$ is any real function.

Then we have

$$
\int_{|\alpha|>A}|V(\alpha)|^{3} K_{v}(\alpha) d \alpha \leq \frac{23}{A} \int_{-\infty}^{\infty}|V(\alpha)|^{3} K_{v}(\alpha) d \alpha
$$

for any $A>4$.

The following inequality holds.

Lemma 6.2 We have

$$
\int_{\mathfrak{c}} \prod_{i=1}^{9} F_{i}\left(\lambda_{i} \alpha\right) G(-\alpha) e\left(-\frac{1}{2} \alpha\right) K_{\frac{1}{2}}(\alpha) d \alpha \ll X^{\frac{121}{60}-6 \delta+\varepsilon} .
$$

Proof We have

$$
\begin{aligned}
\int_{\mathfrak{c}} & \prod_{i=1}^{9} F_{i}\left(\lambda_{i} \alpha\right) G(-\alpha) e\left(-\frac{1}{4} \alpha\right) K_{\frac{1}{4}}(\alpha) d \alpha \\
\ll & \frac{1}{P} \int_{-\infty}^{+\infty}\left|\prod_{i=1}^{9} F_{i}\left(\lambda_{i} \alpha\right) G(-\alpha)\right| K_{\frac{1}{4}}(\alpha) d \alpha
\end{aligned}
$$




$$
\begin{aligned}
\ll & N^{-5 \delta} \max \left|F_{1}\left(\lambda_{1} \alpha\right)\right|^{\frac{1}{5}}\left(\int_{-\infty}^{+\infty}\left|F_{1}\left(\lambda_{1} \alpha\right)\right|^{9}\right)^{\frac{9}{32}}\left(\int_{-\infty}^{+\infty}\left|F_{2}\left(\lambda_{2} \alpha\right)\right|^{9}\right)^{\frac{3}{4}} \\
& \times\left(\prod_{j=3}^{5} \int_{-\infty}^{+\infty}\left|F_{j}\left(\lambda_{j} \alpha\right)\right|^{16} K_{\frac{1}{2}}(\alpha) d \alpha\right)^{\frac{1}{16}}\left(\prod_{k=6}^{8} \int_{-\infty}^{+\infty}\left|F_{k}\left(\lambda_{k} \alpha\right)\right|^{32} K_{\frac{1}{2}}(\alpha) d \alpha\right)^{\frac{1}{32}} \\
& \times\left(\int_{-\infty}^{+\infty}|G(-\alpha)|^{2} K_{\frac{1}{2}}(\alpha) d \alpha\right)^{\frac{1}{2}} \\
\ll & X^{\frac{121}{60}-6 \delta+\varepsilon}
\end{aligned}
$$

from Lemmas 5.1, 6.1 and Schwarz's inequality.

\section{Results}

In this paper, we established that if $\lambda_{1}, \lambda_{2}, \ldots, \lambda_{8}$ are positive real numbers, at least one of the ratios $\lambda_{i} / \lambda_{j}(1 \leq i<j \leq 8)$ is irrational, then the integer parts of $\lambda_{1} x_{1}^{3}+\lambda_{2} x_{2}^{3}+$ $\lambda_{3} x_{3}^{4}+\lambda_{4} x_{4}^{5}+\lambda_{5} x_{5}^{6}+\lambda_{6} x_{6}^{7}+\lambda_{7} x_{7}^{8}+\lambda_{8} x_{8}^{5}$ are prime infinitely often for $x_{1}, x_{2}, \ldots, x_{8}$, where $x_{1}, x_{2}, \ldots, x_{8}$ are natural numbers.

\section{Acknowledgements}

The authors want to thank the reviewers for much encouragement, support, productive feedback, cautious perusal and making helpful remarks, which improved the presentation and comprehensibility of the article. This work was supported by the Higher Education Research Promotion and National Research University Project of Thailand, Office of the Higher Education Commission, through the Cluster of Research to Enhance the Quality of Basic Education.

\section{Competing interests}

The authors declare that they have no competing interests.

\section{Authors' contributions}

JM drafted the manuscript. YW helped to draft the manuscript and revised the written English. Both authors read and approved the final manuscript.

\section{Author details}

${ }^{1}$ Department of Computer Science and Technology, Harbin Engineering University, Harbin, 150001, China. ${ }^{2}$ Faculty of Science and Technology, Tapee College, Surathani, 84000, Thailand.

\section{Publisher's Note}

Springer Nature remains neutral with regard to jurisdictional claims in published maps and institutional affiliations.

Received: 9 December 2016 Accepted: 27 June 2017 Published online: 19 July 2017

\section{References}

1. Danicic, I: On the integral part of a linear form with prime variables. Can. J. Math. 18, $621-628$ (1966)

2. Brüdern, J, Kawada, K, Wooley, T: Additive representation in thin sequences, VIII: Diophantine inequalities in review. Ser. Number Theory Appl. 6, 20-79 (2010)

3. Lai, K, Chiang, Y: Constraint-based fuzzy models for an environment with heterogeneous information-granules. J. Comput. Sci. Technol. 21, 401-411 (2016)

4. Huang, B, Wang, J, Zylbersztejn, M: An extension of the estimation for solutions of certain Laplace equations. J. Inequal. Appl. 2016, 167 (2016)

5. Li, F, Xue, G: Asymptotic behavior of the solution for a diffusive system coupled with localized sources. Dyn. Contin. Discrete Impuls. Syst., Ser. A Math. Anal. 12, 719-730 (2015)

6. Vaughan, R: Diophantine approximation by prime numbers, II. Proc. Lond. Math. Soc. 28, 385-401 (1974)

7. Vaughan, R: The Hardy-Littlewood Method, 2nd edn. Cambridge Tracts in Mathematics, vol. 125. Cambridge University Press, Cambridge (1997)

8. Vaughan, R: Diophantine approximation by prime numbers, I. Proc. Lond. Math. Soc. 28, 373-384 (1974)

9. Davenport, H, Roth, K: The solubility of certain Diophantine inequalities. Mathematika 2, 81-96 (1955) 